\title{
Economic Evaluation of Intergenerational Programs: Suggested Measures and Design
}

\begin{abstract}
Multiple aspects of intergenerational programs present challenges for evaluators. Broader measures and perspectives are needed particularly with respect to informing economic evaluations of intergenerational programs. The study aims to: (1) Identify impact measures suitable for an economic evaluation of a mixed generation program; (2) Design an economic evaluation that compares these program impacts with current services. The suggested evaluation design includes perspectives from children, parents, adult care recipients, caregivers and service providers. Measures for inclusion were based on the literature and selected using a set of criteria and stakeholder interviews. To inform economic evaluations of mixed generation programs we recommend a quasi-experimental design using measures of experience/satisfaction, quality of life/wellbeing, willingness to pay, burden of care and service utilization. The suggested economic evaluation measures and design will guide future programs of mixed generations in the pursuit of cost effective and sustainable service options in this rapidly evolving service environment.
\end{abstract}

Key words: Evaluation; Intergenerational program; Quality of life; Willingness to pay; Quasi-experimental; Measures. 


\section{INTRODUCTION}

The intergenerational revolution continues to gain momentum, in recognition of the need to ensure childcare programs are financially sustainable, better support carers, reduce social fragmentation, improve child development and the quality of life of older people. Indeed, intergenerational programs may provide unique benefits which are not available in traditional care settings and are more convenient for the increasing number of adults who care for young children and aging parents (Weeks et al., 2016). These advantages extend to the unique therapeutic benefits of children engaging with older people and indications that this engagement leads to improved social development and fewer behavioral challenges for the children themselves (Heerema \& Chavas, 2017).

Intergenerational programs are based on the belief that the mixing of generations in purposeful activities that are directed at either children or older people, benefits all participants. The psychological and behavioral benefits of these programs for children include improved attitudes towards aging and children's perceptions about older persons (Cummings et al., 2002; Femia et al., 2008; Generations United, 2013; Heyman et al., 2011; Jarrott \& Bruno, 2007). These programs have also been shown to increase social engagement, confidence, and resilience (Femia et al., 2008; Gualano et al., 2018; Hayes, 2003) for both older and younger participants and improved social behavior associated with sharing, helping and cooperating (Dellman-Jenkins et al., 1991).

Research on the life-course theory identifies the importance of adult support structures early in childhood to improve confidence, security and lower the incidence of bullying and antisocial behavior. This may decrease the likelihood of juvenile delinquency for participants (Whitten et al., 2016). 
While the psychological aspects of mixing generations are often reported by agencies, there is little documented research of other outcomes associated with intergenerational interactions such as improved quality of life, cognitive skills and independence that extends beyond the impacts of younger and older participants of the program. Further, often these impacts are not quantifiable, therefore making decisions around program choice, investment and disinvestment problematic.

Economic evaluation generates evidence-based information that allows policy makers and managers to effectively choose programs based on a comparison of alternatives in terms of costs and consequences (Drummond et al., 2005). A crucial component of the comparative analysis is the choice of measures that is used to quantify and, where possible, monetize impacts (Drummond et al., 2005). Recognizing that impacts of intergenerational programs go beyond that of younger and older participants, analysts can implement economic evaluations that capture a wider range of perspectives. This avoids an underestimation of the program's net benefit.

The study reported here is the first to design and identify measures for an economic evaluation of an intergenerational program. We present an economic evaluation design that identifies appropriate measures to determine the impacts of an intergenerational program from various perspectives - children, parents, adult care recipients, caregivers and agencies. This study will assist policy makers and care agencies in future evaluations for the purpose of promoting cost effective and sustainable care options.

\section{LITERATURE REVIEW}

\section{Intergenerational Evaluation Literature}


A review of the literature by Gualano and colleagues (Gualano et al., 2018) identified ten studies that evaluated children's outcomes from intergenerational programs, outlining the positive impact upon children's perception of elderly. The effects on older participants were varied when considering well-being, depression, self-reported health, and self-esteem. The staff involved in similar programs appeared satisfied. Mosor et al (Mosor et al., 2019) documented well-being among participants as measured by observing spontaneous intergenerational contacts and facial expressions. Evaluation studies such as these (e.g. see (Gualano et al., 2018; Mosor et al., 2019) generally include pre and post data collection points without a comparator, are often confined to direct participants, and use measures that are difficult to quantify such as subjective measures of video observations (Hayes, 2003; Mosor et al., 2019) which are vulnerable to bias (Hayes, 2003).

Researchers and practitioners acknowledge the need for evidence informed evaluation tools to demonstrate the impact of Intergenerational programs on both older and younger participants (Hayes, 2003; Jarrott et al., 2019; Mosor et al., 2019). According to Hayes (2003) much of the shortcoming of intergenerational evaluations can be addressed by ensuring that the instruments utilized for evaluation purposes contain a higher degree of objectivity. Hayes (2003) also highlight the critical importance in developing longitudinal studies to determine intergenerational program outcomes. Capturing the insights of the child/elder caregiver is also important since they may best understand and observe how intergenerational programs influences the participant within the home and other settings (Hayes, 2003).

\section{Economic Evaluation Literature}

The lack of economic evaluations of intergenerational programs is not surprising given the relatively recent introduction of these programs into formal care. There are, however, several economic evaluations that separately document outcomes of programs directed at either 
children or older people. Cost effectiveness is often used to evaluate early childhood [see review by (Dalziel et al., 2015)] or older care programs (Church et al., 2011; Golsteijn et al., 2014; van Leeuwen et al., 2015) [see review by (Knapp et al., 2013)]. These evaluations compare the relative costs and non-monetized outcomes. In contrast, cost benefit analysis is a more comprehensive assessment method that quantifies in monetary terms the value of all impacts (costs and outcomes) (Boardman et al., 2014).

\section{Economic Evaluation of early childhood programs}

Several cost benefit analyses are reported in the literature regarding early childhood programs. A review by Dalziel et al (Dalziel et al., 2015) concluded that early childhood education programs can have a positive impact on cognitive skills, school achievement, social and emotional development, educational achievement, employment and crime. The cost-benefit results consider six center-based early childhood education programs targeted at disadvantaged groups. According to the authors, the design of the trial is important in considering generalizability of study results (Dalziel et al., 2015). A broader scope of included benefits and longer follow-up generally result in a more favorable Cost Benefit ratio with additional benefits being accrued beyond the costs overtime (Dalziel et al., 2015).

Similarly, additional benefits accruing beyond the costs is observed for the Perry Preschool cost benefit studies, with successive studies that incorporate longer follow-up (up to age 40) reporting successful higher cost benefit ratios (Barnett, 1993; Barnett \& Escobar, 1990; Belfield, Nores, Barnett, \& Schweinhart, 2008). The analysis of the Sure State program by Meadows (Meadows, 2011) also reported a larger impact and lower cost when measuring the community-level effects rather than the program participant effects alone. Meadows (Meadows, 2011) notes a number of small effects which may translate into measurable downstream benefits. 


\section{Economic Evaluation of older adult programs}

In contrast to the evaluations of early childhood interventions, the economic evaluations of respite interventions for older adults have produced mixed results. For instance, Mason's review of 37 reference databases of both randomized and quasi experimental studies found no reliable evidence that respite care delays entry to residential care or adversely affects frail older people (Mason et al., 2007). Care giver satisfaction was generally higher than the control groups. The economic evaluations of day care tended to be associated with similar or higher costs than usual care. The existing evidence base did not allow any firm conclusions about effectiveness or cost-effectiveness to be drawn and was unable to inform current policy and practice. The authors (Mason et al., 2007) concluded that there was serious problems with the methodological quality of many studies underpinning the evidence base and where betterquality evidence did exist, the implications for other populations were unclear.

Since respite is a complex intervention with many moving parts, evaluation of respite care using conventional randomized control trials can be challenging (Mason et al., 2007). Recruitment challenges are posed by existing access methods and the diversity of the study population (Mason et al., 2007). Pilot studies could identify one or more target groups (i.e., caregiver and care receiver dyads) suitable for study, establish clear definitions of the services to be compared, and determine the main outcomes to be measured, such as caregiver quality of life or institutionalization rate. The way the questions are framed or respondents' expectations of how the information they provide will be used may bias self-reported evidence from qualitative research. The quality and breadth of data analyzed limit regression analyses of observational data sets, making it difficult to identify true causal effects (Kosloski \& Montgomery, 1995). Because any single approach is unlikely to provide satisfactory data, the merging of different methodologies within a single study may enable qualitative and 
quantitative approaches to compensate for their respective shortcomings. Long-term surveillance could complement findings from these trials.

A review of the literature by Knapp and colleagues (Knapp et al., 2013) found the smaller body of cost-effectiveness evidence on non-pharmacological treatments (cognitive stimulation therapy, tailored activity program and occupational therapy) compared to pharmacological therapies challenging to interpret. Evidence on support for family and other unpaid carers was limited and confined to a narrow range of options: day care, psycho-educational interventions and befriending. Other evidence showed that dementia group sessions in day centers twice a week for 8 weeks improved cognition and quality of life and was more cost effective than usual care (Knapp et al., 2013).

Reflecting the conclusions of Mason's review of the literature (Mason et al., 2007), Knapp and colleagues report similar barriers to achieving better value for money in dementia care that relate to the actual studies themselves (Knapp et al., 2013). One barrier was the scarcity and low methodological quality of the available studies. Among those limitations was the short time frame adopted for measuring most outcomes and costs. Studies reviewed by Mason et al (2007) captured impacts of between 1 and 12 months. Furthermore, generalization from the available evidence was hindered by both the heterogeneity of the populations studied (in terms of severity, co-morbidity, care settings) and familiar inter-country differences in organization, funding and incentives in health and social care systems. Many studies adopted a narrow perspective when measuring cost, looking only at health care. Given the pivotal roles of family and other carers, a broader societal perspective is generally needed if decision makers are to understand the wide range of potential economic impacts, including the opportunity costs of carer inputs and the impacts of caring on their own health and wellbeing.

In a randomized control trial the authors (Knapp et al., 2006) concluded that Cognitive Stimulation Therapy (CST) had the potential to be more cost effective than usual care and 
support through its effects on cognition and quality of life. Costs for people receiving CST were not significantly higher than costs for usual care. These findings were weakened by the small sample and the short follow-up period (8 weeks). As a result, long-term outcomes are not known. The authors note that social interaction between group participants could have generated the positive outcomes rather than the CST itself.

A systematic literature review by Easton (Easton et al., 2016) identified studies that examined dementia-specific structures and processes, in which person-centered interventions demonstrated the potential to reduce agitation and improve residents' quality of life. The review highlighted methodological limitations in the existing evidence and an urgent need for future research to identify appropriate and meaningful outcome measures that can be used at a service planning level.

Vandepitte et al (Vandepitte et al., 2016) described a quasi-experimental study to assess (cost) effectiveness of an in-home respite care program to support informal caregivers of persons with dementia. The primary outcome was caregiver burden. Secondary outcomes included quality of life of caregivers, frequency of behavioral problems of persons with dementia and the reactions of caregivers to those problems, intention to institutionalize the care-recipient, time to nursing home placement, resource use of the care-recipient, and willingness to pay for in-home respite care.

\section{AIM}

Limited health care budgets and population aging have resulted in a need to determine value for money for care programs. Economic evaluations from early childhood and older care programs demonstrate that the impacts are likely to be broader than the psychological aspects currently reported in the literature. A challenge for analysts is the evaluation of intergenerational programs that capture multiple aspects (Hayes, 2003). Thus, broader 
measures are needed to examine their benefits and costs when evaluating these types of programs.

The aims of the study are two-fold:

1. Identify suitable economic evaluation indicators to measure the impacts of an intergenerational program for older people and young children;

2. Design an economic evaluation to compare the costs and consequences of an intergenerational program with the current services offered (separate childcare and respite aged care).

For the purposes of the development of the evaluation design and the identification of appropriate measures a clear understanding of an intergenerational program is important. In this paper an intergenerational care program refers to a specific type of program that involves the caring of older and younger people in a shared setting under the supervision of a formally trained caregiver (Vecchio et al., 2017). The program operates within a community setting. The program aims to benefit older people (and carers) and younger children (for example, 3-5 years) in day care settings. Previous investigation show that a rolling 8 week program works well (Golenko et al., 2020). The program characteristics were conceived from extensive consultations with a panel of experts using a Delphi technique and considered based on the practical and feasible implementation of the program within an Australian setting (Vecchio et al., 2017).

Based on previous research we hypothesize that the intergenerational program has the potential to broadly impact on several key groups (Dalziel et al., 2015; Knapp et al., 2013; Vandepitte et al., 2016). The evaluation design will therefore demonstrate a range of stakeholder perspectives. Adult care recipients may be impacted by an improvement in wellbeing/quality of life, improved social engagement, reduced usage of health care services, improved independence (Knapp et al., 2013). In relation to children, we would expect some improvement 
in social engagement skills (Dalziel et al., 2015). Parents of young children and adult family carers are also expected to be impacted by the program through reduced burden of care and stress levels (Vandepitte et al., 2016). Furthermore, agencies are likely to experience a change in costings, possibly opportunities for reduced operating costs longer-term and improved staff work satisfaction in the short and medium term.

As summarized from the literature, Table 1 lists the hypothesized impacts of an intergenerational program, stratified by perspective. This stylized framework displays the impacts from the perspectives of participants and nonparticipants and suggests some of the distributional implications of the program. Since the measures of impact listed in the table can be obtained from data collected during the evaluation, it is operationally feasible (Boardman et al., 2014). Note that plus signs indicate anticipated sources of benefits and minus signs indicate anticipated sources of costs for different perspectives. ' 0 ' indicates no change. The last column shows aggregate benefits and costs from the perspective of the society. The other columns describe the anticipated distribution of benefits and costs to the groups of participants in the programs and their care givers. Impacts to society are the algebraic sum of benefits and costs.

\section{[INSERT TABLE ONE HERE]}

\section{MEASURES}

Careful consideration was given to the identification and selection of measures appropriate in capturing the multiple impacts of the program listed in Table 1. Selection was based on a set of criteria and informed by the literature. Considerations included published recommendations, perspectives, agency setting (e.g. child-care center, aged care home, respite community center), childcare and aged care sector and timeframe. 


\section{Selection of economic evaluation measures}

Inclusion of key measures to capture the impacts of an intergenerational program are based on four key criteria: data availability; ease of monetization; reliability; and validity.

In economic evaluations the choice of indicators to measure the impacts primarily depend on data availability and ease of monetization (Boardman et al., 2014). To increase the likelihood of available data, measures that are simple to administer, collect and user friendly are given preference. Measures collected and recorded within the current domain/job are also preferred over those that require additional work for agency staff.

Monetization translates qualitative impacts into one globally understood metric, money, so that stakeholders can accurately compare outcomes across different scenarios. Where possible generic measures are preferred due to their transferability and ease of comparison across other programs and studies (and relative ease of their implementation). There is greater potential for generic measures to be monetized. While cost benefit analysis requires the monetization of all impacts, it is not always possible or practical to do so. This often leads analysts to perform other forms of economic evaluations such as cost effectiveness analysis.

Choice of measures also take into consideration the reliability and validity of the measure. A reliable measure produces similar results when applied under consistent conditions. Validity is the extent to which a tool measures what it is supposed to measure. The measurement tools need to be effective in collecting the impact category. Only those that are essential to the end goal are considered. There is also a preference for measures that have the potential to detect change via modifiable indicators.

\section{Description of the selected economic evaluation measures}

Table 2 presents suggested measures that capture the multiple impacts of an Intergenerational program. Given varying environmental, political, cultural and economic factors, an analyst 
must often use discretion when choosing measures. The list of measures discussed below is not exhaustive and should be viewed as a guide.

[INSERT TABLE 2 HERE]

WHO-5 Well-Being Index (1998 version)

Regarding measures of well-being and quality of life, the WHO-5 Well Being Index was chosen over the WHOQoL-BREF (Standard) and the EQ-5D. The 5-item Index is among the most widely used questionnaires assessing subjective psychological well-being. The instrument consists of simple and non-invasive questions, which tap into the subjective wellbeing of the respondents (Topp et al., 2015). A major consideration was the simplicity of the instrument.

This measure is administered to parents of children, care recipients and care givers of adults. In this measure participants indicate each of the five statements which is closest to how they have been feeling over the last two weeks. The higher numbers mean better well-being. The raw score is calculated by totaling the figures of the five answers and ranges from 0 to 25,0 representing worst possible and 25 representing best possible quality of life. To obtain a percentage score ranging from 0 to 100 , the raw score is multiplied by 4 . A percentage score of 0 represents worst possible, whereas a score of 100 represents best possible quality of life. A score below 13 indicates poor wellbeing and is an indication for testing for depression under ICD-10. In order to monitor possible changes in wellbeing, the percentage score is used. A $10 \%$ difference indicates a significant change (ref. John Ware, 1995).

The scale has adequate validity both as a screening tool for depression and as an outcome measure in clinical trials and has been applied successfully across a wide range of study fields. 
Item response theory analyses in studies of younger persons and elderly persons indicate that the WHO-5 measure has good construct validity as a unidimensional scale measuring wellbeing in these populations (Topp et al., 2015).

\section{The Leuven Well-being and Involvement scales}

The tool is intended for children and focuses on two central indicators of quality early years provision: children's 'well-being' and 'involvement'. Well-being refers to feeling at ease, being spontaneous and free of emotional tensions and is crucial to good 'mental health'. Wellbeing is linked to self-confidence, a good degree of self-esteem and resilience. Involvement refers to being intensely engaged in activities and is a necessary condition for deep level learning and development. The five-point scale measures both well-being and involvement. The higher the levels of well-being and involvement that can be achieved for the child, the more can be added to the child's development including a deeper level of learning. The staff member observes the children individually or as a group for about two minutes then gives a score for wellbeing and/or involvement using the five-point scale (Laevers et al., 2005).

The Leuven Well-being and Involvement scales are preferred over other possible measures of well-being for children such as the Social Capital Scale (Onyx \& Bullen, 1998). The scales are internationally recognized as an outcome measure (Ebbeck et al., 2012; Laevers \& Declercq, 2018). The Leuven scales are currently collected and recorded by some agencies in the Australian childcare sector. This means that data is easily available with request for data requiring little or no additional work by agency staff. The measure procedure is simple to administer and collect. Access to user friendly on-line resources adds to the attractiveness of this measure. The wide use of the scales allows transferability and ease of comparison.

Kingston Caregiver Stress Scale (KCSS) 
KCSS is designed to allow a family caregiver to express the level of stress that he or she is currently feeling around the duties of care. KCSS consists of a set of ten questions. Using a five-point rating scale, where 1 equals no stress and 5 equals extreme stress, indicate the extent of the stress or frustration that the care giver feels surrounding care giving, family and financial issues (Hopkins et al., 2007).

The measure was constructed in response to evidence that other carer scores did not correlate well with how much stress an individual caregiver experienced. Validity of KCSS is improved from previous measures (Hopkins et al., 2007). The scale is designed for community living family caregivers, rather than institutional care staff. The KCSS takes a more direct approach by asking how much stress the caregiver feels. The scale can also be used to monitor change in an individual caregiver's stress over time. Evidence suggests that caregivers can attribute their stress to different sources which is useful for the purposes of evaluation. A strength of this measure is the recognition and inclusion of different sources of stress - care giving, family, and financial.

\section{Life Orientation Test-Revised (LOT-R)}

LOT-R is a revised version of the original LOT (Scheier et al., 1994). The revised measure is a 10-item measure of optimism versus pessimism. Of the 10 items, 3 items measure optimism, 3 items measure pessimism, and 4 items serve as fillers. Respondents rate each item on a 4point scale: $0=$ strongly disagree, $1=$ disagree, $2=$ neutral, $3=$ agree, and $4=$ strongly agree

The Life Orientation Test has been shown to possess adequate predictive and discriminant validity. Associations between optimism and both depression and aspects of coping remain significant even when the effects of neuroticism, trait anxiety, self-mastery, and self-esteem, are statistically controlled (Scheier et al., 1994). The LOT-R can be used as a reliable and valid 
tool for analysis of optimism and pessimism and its effect on health and cognitive and emotional attributes (Gustems-Carnicer et al., 2017).

ASCOT

The ASCOT measure is designed to capture information about an individual's social carerelated quality of life (SCRQoL). The measure is applicable across as wide a range of user groups and care and support settings. In identifying and defining the domains, the measure is sensitive to outcomes of social care related quality of life. Using the current version of ASCOT, outcomes can be measured in several ways such as to measure changes over time in 'current' SCRQoL. The SCT4 is a four-level self-report version that measures current SCRQoL in community settings. This is the version proposed in the evaluation for care recipients (see Table 2). The questionnaire considers statements describing someone's personal situation in multiple aspects of their life such as personal cleanliness, and comfort, accommodation cleanliness and comfort, food and drink, safety, social participation and involvement, occupation, control over daily life and dignity.

The ASCOT is designed specifically for evaluating interventions in social care and can potentially be applied in many sectors such as social care and institutionalized care (Hackert et al., 2017). The advantage of ASCOT for evaluation purposes is its ability to capture the expected change in quality of care (Netten et al., 2012). The introduction of ASCOT by the Australian Department of Health as a measure for use in aged care services gives further weight to using this measure in an evaluation. The ASCOT index has good test-retest reliability for adults with physical or sensory disabilities who use social care services (Rand et al., 2017). There is evidence for the validity of the ASCOT attributes and therefore support for ASCOT's use in economic evaluations (Malley et al., 2012). Feasibility of its use among older people has also been demonstrated (Malley et al., 2012). 


\section{Satisfaction Scale}

The three versions of the Satisfaction scale for the evaluation include: Parent, Care recipient and Care giver. The Resident Satisfaction Questionnaire is a self-administered questionnaire covering five service areas (case management, adult day care in respite and neighborhood centers, in-home respite, emergency in-home counseling and overnight crisis care) and previously used to document resident satisfaction in aged care (Chou et al., 2001). The Resident Satisfaction Questionnaire is confirmed to be a reliable, valid, context-relevant, and easy-to-use instrument for assessing residents' satisfaction with their residential aged care facilities (Chou et al., 2001). The scale is useful in economic evaluations in determining which scenario of programs provide the greatest level of satisfaction.

\section{Willingness to Pay}

Surveys of population samples can be used as the basis for estimating aggregate willingness to pay for a program. This approach, called 'contingent valuation,' is conceptually attractive because it potentially allows the analyst to elicit a willingness-to-pay amount for programs that involve both active and passive use (Haveman \& Weimer, 2001). This measure is used extensively in cost benefit analysis. To ascertain the value that individuals place on the service the evaluation includes a willingness to pay question. A higher willingness to pay, holding all else constant, indicates that the participant places a higher value on this service. This stated preference approach elicits a monetary value to a care program. Studies demonstrate the validity and reliability of this measure under various contexts (Amoah et al., 2019; De Meijer et al., 2010; Loomis, 1989; Settumba et al., 2019).

\section{Health Services}

The use of health services is often used in economic evaluations to determine the impact of a program on older participants (Golsteijn et al., 2014; Leung et al., 2004; van Leeuwen et al., 
2015; Weimer \& Sager, 2009). This can be measured by asking: 'Do you currently have any pre-existing health conditions?' and 'How many times in the last 2 weeks did you visit a Health Professional?' This data is easily collected and converted to a monetary value.

\section{Curbow Job Stress Scales}

The Curbow job stress inventory for child care workers provides a tool that can be used to: compare child care environments; track stressor levels in workers over time; assess the spillover effects of stressors from one group in the network to another; and to suggest possible interventions that might allay the effects of work place stressors. Embedded in the instrument are three job stress scales, specific to child care workers, measuring job demands, job control, and job resources (Curbow et al., 2000). The 5-point rating scale asks how often each statement happens at work. The reliability and convergent and discriminant validity of the scales have been tested for both family child care provider and center-based teacher samples (Curbow et al., 2000).

\section{METHOLDOGY}

\section{Economic evaluation design}

Economic evaluations of any intervention require comparisons between alternatives through which the program can be compared to the situation that would exist without the program. The impacts are measured as differences in the outcomes between the two scenarios.

Randomized control trials while offering high internal validity tend to have poor external validity, which means results are unlikely to be replicated in population rollout. On the contrary, community evaluations (such as Sure Start (Meadows, 2011)) based on nonrandomized trials provide an accurate picture of what a scaled up program would look like, but they often suffer from internal validity constraints in demonstrating effectiveness (Dalziel et 
al., 2015). Thus evaluations that provide an understanding of a scaled up version of the program but lack internal validity may be preferred over randomized control trials that offer poor external validity (Dalziel et al., 2015).

For practical reasons we propose that the economic evaluation of an intergenerational care program uses a quasi-experimental design that involves the non-random allocation of participants into the control and intervention groups. A nonexperimental comparison is made with baseline data (Boardman et al., 2014). Both pre-intervention and post-intervention data are collected. This provides information on how the intervention group differs from the comparison group prior to the program. This information can be used in a statistical analysis control for pre-intervention differences between the intervention and control groups. Given the changes that we are likely to see in a child's development overtime regardless of the intervention, the importance of the control group is apparent. We can also expect to see changes in the older adults over time, in both their physical and mental health. The control group therefore assists in determining if the changes are the result of the program or due to natural changes that might occur among the different groups overtime.

Although this evaluation design offers greater opportunity to obtain internal validity than other nonrandomized designs (Boardman et al., 2014), it may lead to selection bias. To reduce this bias and improve internal validity, several measures can be taken to ensure that the characteristics of the control and intervention groups are similar. For instance, it is not unusual for an organization to operate several child-care or aged care/respite centers. Deriving the control groups and intervention groups from the same organization and matching intervention groups with control groups that have similar characteristics and demographics will improve internal validity. The control and intervention group derived from the same organization are likely to have similar staff culture, procedures, funding arrangements and resources. 


\section{Timeline Intervention}

Although previous research of Intergenerational programs has observed positive change in select participants, studies recommend that evaluations track change and activity outcomes over time (Dalziel et al., 2015; Hayes, 2003; Knapp et al., 2013). An investigation over several years is more likely to capture impacts among older adults such as service usage and nationalization rates, as well as impacts among children such as educational attainment. However, funding or resource constraints often determine the timeframe for the investigation of impacts.

As the evaluation timeline increases, we can expect an increase in benefits (Dalziel et al., 2015). Regarding costs, as the timeframe is extended unit costs are expected to decrease. This is mainly the result of fixed costs spread over a longer timeline. The timeframe limits the evaluation to private impacts and reduces the prospects of capturing social impacts. Long-term surveillance could complement findings from trials. (Kosloski \& Montgomery, 1995). Sensitivity analysis can be used to calculate total costs when the timeframe changes.

Measurement tools should, at a minimum, be collected at base line and end point. A relatively short time frame of 16 weeks may be feasible and takes into consideration industry fatigue, time and resource constraints in the collection of the information.

\section{Comparison groups and perspectives}

It is expected that the impact of the programs be reported from the perspective of several key groups: older people receiving day care; preschool age children (3 to 5 years); parents; family care givers; and agencies.

Benefits and costs of the intergenerational program (intervention) are compared to the status quo (e.g. separate childcare and aged care). The economic evaluation can incorporate two major categories of analysis: a. Care of children where costs and benefits of the 
intergenerational program are compared with the impacts of a traditional childcare center. This evaluation can be analyzed from the perspective of the child, parent and agency; b. Care of adults where costs and benefits of the intergenerational program are compared with the impacts of a traditional aged care center. This evaluation can be analyzed from the perspective of the adult, care giver and agency.

\section{Data Sources}

The collection of information from a single approach can be inadequate (Kosloski \& Montgomery, 1995). It is therefore likely that economic evaluations on intergenerational programs will need to supplement primary data with information collected from industry (e.g. annual report, attendance patterns, staff turnover, budgets), published literature and reports.

\section{Monetization of impacts}

We expect that some of the benefits may be difficult or unable to be monetized. Even so, excluding these impacts from the analysis implies that they have no value when in fact they may be influential to the overall effectiveness of the program. Qualitative impacts such as increased positive attitudes, friendships can be discussed and ranked in order of magnitude and importance. In some instances, a cost effectiveness analysis may be performed for outcomes that are difficult to monetize.

\section{Sensitivity Analysis}

An essential component of an economic evaluation is the sensitivity analysis. A sensitivity analysis is typically conducted on uncertain information that is expected to have a considerable and likely influence on the Net Present Value (NPV) of the program (Boardman et al., 2014). When NPV is positive, the program should proceed. Uncertainty may include information around the actual take up of the program or outcomes of the program. 
Sensitivity analysis is particularly useful to agencies seeking to identify the best case scenario (Boardman et al., 2014) among mixes of resources and staffing options. The business implication of changing cost items, regulations and ratios is also important to agencies. For example, an agency can obtain an understanding of the viability of a program (impact on the NPV) when wages increase/decrease by $5 \%$ or staff child ratios rise/fall substantially or staff turnover changes substantially.

Another consideration that can be incorporated into the sensitivity analysis is the probability of certain risk factors (such as injury or infection) occurring as a result of the program (Boardman et al., 2014). Such analysis can identify the consequences of these risk events from a range of perspectives including agencies and clients.

\section{CONCLUSION}

Based on the literature, a stylized economic evaluation framework was used to present the hypothesized impacts of an intergenerational program. Careful consideration was given to the identification and selection of measures appropriate in capturing the multiple impacts of the program. The measures discussed are clearly not exhaustive. They do, however, provide guidance for future economic evaluation work. Key measures for inclusion in the evaluation were based on the literature and interviews with expert stakeholders. Measures were refined in accordance with identified criteria to ensure they were appropriate to the end goal, had the potential to detect change and were simple to administer and to collect the required data. Generic measures were preferred due to transferability and ease of comparison across other programs and studies.

The primary outcome measures included experience/satisfaction of the program, quality of life/wellbeing and willingness to pay. From the care giver and staff perspective, measures included burden of care and job stress (respectively). From the care recipient perspective, the 
measures included use of health services. Information on the marginal costs of intervention from the perspective of the agencies was also considered in the context of the business case of the program. A quasi-experimental design elicits perspectives from children, parents, adult care recipients, caregivers and service providers. Considerations included feasibility of the design, published recommendations, perspectives, agency setting, childcare and aged care sector and timeframe.

There is currently little evidence that documents the multiple impacts of Intergenerational programs. This study took initial steps to fill this knowledge gap by identifying appropriate measures that can be used to perform economic evaluations of mixed generation programs. The design considers stakeholder needs as well as some emerging ideas in relation to feasible strategies to manage potential sources of bias. The economic evaluation design and measures will guide future programs of mixed generations in the pursuit of cost effective and sustainable service options in this rapidly evolving social, economic and business environment.

\section{REFERENCES}

Amoah, A., Ferrini, S., \& Schaafsma, M. (2019). Electricity outages in Ghana: Are contingent valuation estimates valid? Energy Policy, 135.

Boardman, A., Greenberg, D., Vining, A., \& Weimer, D. (2014). Cost Benefit Analysis. Concepts and Practice. Fourth Edition. Pearson.

Bulamu, N., Kaambwa, B., \& Ratcliffe, J. (2015). A systematic rview of instruments for measuring outcomes in economic evaluation within aged care. Health and Quality of Life Outcomes, 13(179).

Chou, S., Boldy, D., \& Lee, A. (2001). Measuring resident satisfaction in residential aged care. The Gerontologist, 41(5), 623-631.

[Record \#35 is using a reference type undefined in this output style.]

Cummings, S., Williams, M., \& Ellis, R. (2002). Impact of Intergenerational Program on 4th Graders Attitudes Towards Elders And School Behaviors. Journal of Human Behavior in the Social Environment, 6(3), 99-107.

Curbow, S., Spratt, K., Ungaretti, A., McDonnell, K., \& Breckler, S. (2000). Development of the child care worker job stress inventory. Early Childhood Research Quarterly, 15(4), 515-536. <Go to |S|>://WOS:000168106400005

Dalziel, K., Halliday, D., \& Segal, L. (2015). Assessment of the cost-benefit literature on early childhood education for vulnerable children: What the findings mean for policy Sage Open, 1-14. 
De Meijer, C., Brouwer, W., Koopmanschap, M., Van Den Berg, B., \& Van Exel, J. (2010). The value of informal care-a further investigation of the feasibility of contingent valuation in informal caregivers. Health Economics, 19, 755-771. https://doi.org/10.1002/hec.1513

Dellman-Jenkins, M., Lambert, D., \& Fruit, D. (1991). Fostering Pre-schoolers' Pro-social Behaviors Toward The Elderly: The Effect Of An Intergenerational Program. Educational Gerontology, $17,1$.

Drummond, M., Sculpher, M., Torrance, G., O'Brien, B., \& Stoddart, G. (2005). Methods for the Economic Evaluation of Health Care Programmes (3 ed.) Oxford University Press.

Easton, T., Milte, R., Crotty, M., \& Ratcliffe, J. (2016). Advancing aged care: a systematic review of economic evaluations of workforce structures and care processes in a residential care setting. Cost effectiveness and resource allocation, 12(14), 1-19. https://doi.org/DOI 10.1186/s12962-016-0061-4

Ebbeck, M., Winter, P., Russo, S., Yin Bonnie Yim, H., Lian Choo Teo-Zuzarte, G., \& Goh, M. (2012). Measuring children's involvement as an indicator of curriculum effectiveness: a curriculum evaluation of a selected child study centre in Singapore. Early Child Development and Care, 182(5), 609-619. https://doiorg.libraryproxy.griffith.edu.au/10.1080/03004430.2011.566329

Femia, E., Zarit, S., Blair, C., \& Bruno, K. (2008). Intergenerational preschool experiences and the young child: Potential benefits to development. Early Childhood Research Quarterly, 23(2), 272-287. https://doi.org/DOI: 10.1016/j.ecresq.2007.05.001

Generations United. (2013, 28/1/2014). America's Best Intergenerational Communities: Building Livable Communities for Children, Youth, Families, and Older Adults Washington DC. www.gu.org

Golenko, X., Radford, K., Fitzgerald, J., Vecchio, N., Cartmel, J., \& Harris, N. (2020). Uniting generations: A research protocol examining the impacts of an intergenerational learning program on participants and organisations. Australasian Journal on Ageing. https://doi.org/10.1111/ajag.12761

Golsteijn, R., Peels, D., Evers, S., Bolman, C., Mudde, A., de Vries, H., \& Lechner, L. (2014). Costeffectiveness and cost-utility of a Web-based or print-delivered tailored intervention to promote physical activity among adults aged over fifty: An economic evaluation of the Active Plus intervention. International Journal of Behavioural Nutrition and Physical Activity, 11, 117.

Gualano, M., Voglino, G., Bert, F., Thomas, R., Camussi, E., \& Siliquini, R. (2018). The impact of intergenerational programs on children and older adults: A review. International Psychogeriatrics, 04/2018, Volume 30, Issue 4, 30(4).

Gustems-Carnicer, J., Calderón, C., \& Santacanab, M. (2017). Psychometric properties of the Life Orientation Test (LOT-R) and its relationship with psychological well-being and academic progress in college students. Revista Latinoamericana de Psicología, 49(1), 19-27. https://doi.org/10.1016/j.rlp.2016.05.001

Hackert, M., Exel, J., \& Brouwer, W. (2017). Valid outcome measures in care for older people: Comparing the ascot and the ICECAP-O. Value in Health 20, 936-944.

Haveman, R., \& Weimer, D. (2001). Cost-Benefit Analysis International Encyclopedia of the Social \& Behavioral Sciences. https://www.sciencedirect.com/topics/economics-econometrics-andfinance/willingness-to-pay

Hayes, C. (2003). An Observational Study in Developing an Intergenerational Shared Site Program. . Journal of Intergenerational Relationships, 1(1), 113-132.

Heerema, \& Chavas. (2017, 3 December, 2019). Therapeutic benefits of children for people living with dementia 
Heyman, J., Gutheil, I., \& White-Ryan, L. (2011). Preschool Children's Attitudes Toward Older Adults: Comparison of Intergenerational and Traditional Day Care. Journal of Intergenerational Relationships, 9, 435-444.

Hopkins, R., Kilik, L., \& Day, D. (2007). Kingston Caregiver Stress Scale Administration and Interpretation Manual. http://www.providencecare.ca/wp-content/uploads/2016/10/KCSSAssessment-Form.pdf

Jarrott, S., \& Bruno, K. (2007). Shared site intergenerational programs: A case study. Journal of Applied Gerontology, 26(3), 239-257.

Jarrott, S., Juckett, L., \& Naar, J. (2019). Increasing the power of Intergenrational networks: Advancing a new evaluatin tool. Innovation in Aging, 3, S672.

Knapp, M., lemmi, V., \& Romeo, R. (2013). Dementia care costs and outcomes: A systematic review. International Journal of Geriatric Psychiatry, 28, 551-561.

Knapp, M., Thorgrimsen, L., Patel, A., Spector, A., Hallam, A., Woods, B., \& Orrell, M. (2006). Cognitive stimulation therapy for people with dementia: costeffectiveness analysis. $\mathrm{Br} J$ Psychiatry 188, 574-580.

Kosloski, K., \& Montgomery, R. (1995). The impact of respite use on nursing home placement. Gerontologist 35, 67-74.

Laevers, F., Daems, M., De Bruyckere, G., Declercq, B., Moons, J., Silkens, K., Snoeck, G., \& Van Kessel, M. (2005). Well-being and Involvement in Care Settings. A Process-oriented Selfevaluation Instrument.

Laevers, F., \& Declercq, B. (2018). How well-being and involvement fit into the commitment to children's rights. European Journal of Education, 53, 325-335. https://doi.org/DOI: 10.1111/ejed.12286

Leung, A. C., Liu, C., Chow, N., \& Chi, I. (2004). Cost-Benefit analysis of a case managment project for the community dwelling frail elderly in Hong Kong. The journal of applied gerontology, 23(1), 70-85.

Loomis, J. (1989). Test-retest reliability of the contingent valuation method: A comparison of general population and visitor responses. American Journal of Agricultural Economics, 71(1), 76-84. https://doi.org/DOI: 10.2307/1241776

Makai, P., Brouwer, W., Koopmanschap, M., Stolk, E., \& Nieboer, A. (2014). Quality of life instruments for economic evaluatoins in health and social care for older people: A systematic review. Social Science \& Medicine, 102, 83-93. https://doi.org/10.1016/j.socscimed.2013.11.05

Malley, J., Towers, A., Netten, A., Brazier, J., Forder, J., \& Flynn, T. (2012). An assessment of the construct validity of the ASCOT measure of social care-related quality of life with older people. Health and Quality of Life Outcomes, 10.

Mason, A., Weatherly, H., Spilsbury, K., Golder, S., Arksey, H., Adamson, J., \& Drummond, M. (2007). The effectiveness and cost-effectiveness of respite for caregivers of frail people. The American Geriatrics Society, 55(2), 290-299. https://doi.org/10.1111/j.15325415.2006.01037.x

[Record \#28 is using a reference type undefined in this output style.]

Mosor, E., Waldherr, K., Kjeken, I., Omara, M., Ritschl, V., Pinter-Theiss, V., Smolen, J., Hübel, U., \& Stamm, T. (2019). An intergenerational program based on psycho-motor activity promotes well-being and interaction between preschool children and older adults: results of a process and outcome evaluation. BMC Public Health, 19(1).

Netten, A., Burge, P., Malley, J., Potoglou, D., Towers, A., Brazier, J., Flynn, T., Forder, J., \& Wall, B. (2012). Outcomes of Social Care for Adults: Developing a Preference-Weighted Measure. Health Technology Assessment, 16(16), 1-165. https://doi.org/http://dx.doi.org/10.3310/hta16160

Onyx, \& Bullen. (1998). Social Capital Scale. 
Rand, S., Malley, J., Towers, A., Netten, A., \& Forder, J. (2017). Validity and test-retest reliability of the self-completion adult social care outcomes toolkit (ASCOT-SCT4) with adults with longterm physical, sensory and mental health conditions in England. Health and Quality of Life Outcomes, 15(163).

Scheier, M., Carver, C., \& Bridges, M. (1994). Distinguishing optimism from neuroticism (and trait anxiety, self-mastery, and self-esteem): a reevaluation of the Life Orientation Test. J Pers Soc Psychol, 67(6), 1063-1078.

Settumba, S., Shanahan, M., Botha, W., Ramli, M., \& Chambers, G. (2019). Reliability and validity of the contingent valuation method for estimating willingness to pay: A case of in vitro fertilisation. Appl Health Econ Health Policy, 17(1), 103-110. https://doi.org/doi: 10.1007/s40258-018-0433-3

Topp, C. W., Østergaard, S. D., Søndergaard, S., \& Bech, P. (2015). The WHO-5 Well-Being Index: A systematic review of the literature. Psychotherapy and Psychosomatics, Psychotherapy and Psychosomatics(84), 167-176.

van Leeuwen, K., Bosmans, J., Jansen, A., Hoogendijk, E., Muntinga, M., van Hout, H., Nijpels, G., van der Horst, H., \& van Taulder, M. (2015). Cost-effectiveness of a chronic care model for frail older adults in primary care: Economic evaluation alongside a stepped-wedge clusterrandomised trial. The American Geriatrics Society, 63, 2494-2504.

Vandepitte, S., Noortgate, N., Putman, K., Verhaeghe, S., \& Annemans, L. (2016). Effectiveness and cost-effectiveness of an in-home respite care program in supporting informal caregivers of people with dementia: design of a comparative study. BMC Geriatrics, 16.

Vecchio, N., K., R., Fitzgerald, J., Comans, T., Harris, P., \& N., H. (2017). Intergenerational care: an exploration of consumer preferences and willingness to pay for care. Aging and Mental Health.

Weeks, L., MacQuarrie, C., Begley, L., Nilsson, T., \& MacDougall, A. (2016). Planning an intergenerational shared site: Nursing-home staff perspectives. Journal of Intergenerational Relationships, 14(4), 288-300. https://doi.org/doi.org/10.1080/15350770.2016.1229550

Weimer, D., \& Sager, M. (2009). Early identification and treatment of Alzheimer's disease: Social and fiscal outcomes. Alzheimer's and Dementia, 5, 215-226.

Whitten, T., Vecchio, N., Radford, K., \& Fitzgerald, A. (2016). Intergenerational Care as a Viable Intervention Strategy for Children at Risk of Delinquency Australian Journal of Social Issues. 